The modern literature suggests that endoscopic resection is adequate in the treatment of this tumor. Submucosal esophageal cavernous hemangiomas have been successfully resected by the endoscopic polypectomy or mucosal resection techniques. ${ }^{4,5}$ Alternatively, other ablative techniques, such as laser therapy, also have been described in the treatment of cavernous hemangiomas. ${ }^{3}$ These modalities, however, are used in the treatment of lesions that are smaller than the one described in the present patient.

Before modern endoscopic instrumentation, limited local resection in the form of a polypectomy or enucleation was the accepted or even favored method of treatment. Approaches to a limited resection have classically been through a thoracotomy. ${ }^{2}$ Recently, thoracoscopic removal has also been described in the treatment of these lesions with the simultaneous use of a flexible endoscope. $^{2}$ This obviously is possible only if the lesion is small enough for this type of resection. Esophagectomy has rarely been described in the treatment of this lesion, but this is because, in general, the majority of cavernous hemangiomas of the esophagus reported are on the order of 2 to $3 \mathrm{~cm} .^{3}$ The hemangioma described in this report was giant, and concerns of malignancy and potential hemorrhage warranted an esophagectomy.

\section{References}

1. Feist K, Talley NA, Hunt DR. Hemangioma of the esophagus: an unusual cause of upper gastrointestinal bleeding. JAMA. 1976;235:1146-7.

2. Ramo OJ, Salo JA, Bardini R, Nemlander AT, Farkkila M, Mattila SP. Treatment of submucosal hemangioma of the esophagus using a simultaneous video-assisted thoracoscopy and esophagoscopy: description of a new minimally invasive technique. Endoscopy. 1997;29:S27-8.

3. Shigemitsu K, Naomoto Y, Yamatsuji T, Ono K, Aoiki H, Haisa M, et al. Esophageal hemangioma successfully treated by fulguration using potassium titanyl phosphate/yttrium aluminum garnet (KTP/YAG) laser: a case report. Dis Esoph. 2000;13:161-4.

4. Tominaga K, Arakawa T, Ando K, Umeda S, Shiba M, Suzuki N, et al. Oesophageal cavernous haemangioma diagnosed histologically, not by endoscopic procedures. J Gastroenterol Hepatol. 2000;15:215-9.

5. Cantero D, Yoshida T, Ito M, Suzumi M, Tada M, Okita K. Esophageal hemangioma: endoscopic diagnosis and treatment. Endoscopy. 1994;26: 250-3.

\title{
Primary Ewing sarcoma invading the heart: Resection and reconstruction
}

\author{
Subroto Paul, MD, ${ }^{\text {a }}$ Tharumenthiran Ramanathan, MD, PhD, FRACS, ${ }^{\text {b }}$ Daniel M. Cohen, MD, a Abraham Lebenthal, MD, ${ }^{a}$ \\ Lambros Zellos, MD, ${ }^{a}$ Sary F. Aranki, MD, and David J. Sugarbaker, MD, ${ }^{a}$ Boston, Mass
}

\section{Clinical Summary}

$\mathrm{W}$ e describe the case history of a 14-year-old boy who came to his pediatrician with a cough. Chest radiograph revealed a large mediastinal mass. Workup with chest computed tomography (CT) and magnetic resonance imaging (MRI) revealed that the mass had invaded the right pulmonary parenchyma, both atria, and the superior vena cava (SVC) (Figure 1, A). CT-guided biopsy revealed the tumor to be Ewing sarcoma/primitive neuroectodermal tumor. The patient underwent sarcoma-based chemotherapy with 18 weeks of vincristine, ifosafamide, doxorubicin (Adriamycin), and etoposide followed by 2 additional weeks of vincristine, doxorubicin, and isofamide. Repeat imaging revealed the mass to be downsized by chemotherapy

From the Divisions of Thoracic Surgery ${ }^{\mathrm{a}}$ and Cardiac Surgery, ${ }^{\mathrm{b}}$ Department of Surgery, Brigham and Women's Hospital, Boston, Mass.

Received for publication Jan 6, 2007; accepted for publication Feb 7, 2007.

Address for reprints: David J. Sugarbaker, MD, Brigham and Women's Hospital, Division of Thoracic Surgery, 75 Francis St, Boston, MA 02115 (E-mail: dsugarbaker@partners.org).

J Thorac Cardiovasc Surg 2007;133:1667-9

$0022-5223 / \$ 32.00$

Copyright () 2007 by The American Association for Thoracic Surgery doi:10.1016/j.jtcvs.2007.02.003
(Figure 1,B). Bone scan, abdominal CT, and positron emission tomography-CT did not reveal any metastases. The patient was referred for surgery. Preoperative workup included pulmonary function tests (forced expiratory volume in 1 second $=2.59$ [67\%], forced vital capacity $=3.00[67 \%]$ ), a quantitative ventilation/perfusion scan (quantitative perfusion [left lung] = $81 \%$; quantitative perfusion [right lung] $=19 \%$ ), and an echocardiogram (ejection fraction $=65 \%$ with normal left and right ventricular function).

A median sternotomy was performed and both pleurae and the pericardium were entered. The mass involved the distal third of the SVC, the free wall of the right and left atria, the interatrial septum, and the right lung, but was deemed resectable. The patient was placed on cardiopulmonary bypass via aortic and bicaval cannulation and cooled to $28^{\circ} \mathrm{C}$ with the heart arrested with antegrade blood cardioplegia. An en bloc resection of the lower third of the SVC, the free wall of the right and left atria along with the interatrial septum, and a right pneumonectomy were performed (Figure 2, A). Frozen section margins were negative for tumor involvement. The interatrial septum and free wall of the right atrium were reconstructed with glutaraldehyde-treated pericardium (Figure 2, $B$ and $C$ ). The SVC was reconstructed with bovine pericardium fashioned into a tube using a double row of staples (US Surgical Corporation, Norwalk, Conn) (Figure 2,D). The distal end of the graft was anastomosed in an end-to-end fashion to the SVC. The proximal end of the tube graft was anastomosed to a transverse opening in the right atrial pericardial patch. The pericardium was reconstructed with porcine submucosal tissue grafts (Cormatrix; Cormatrix Cardiovascular, Atlanta, Ga) to 


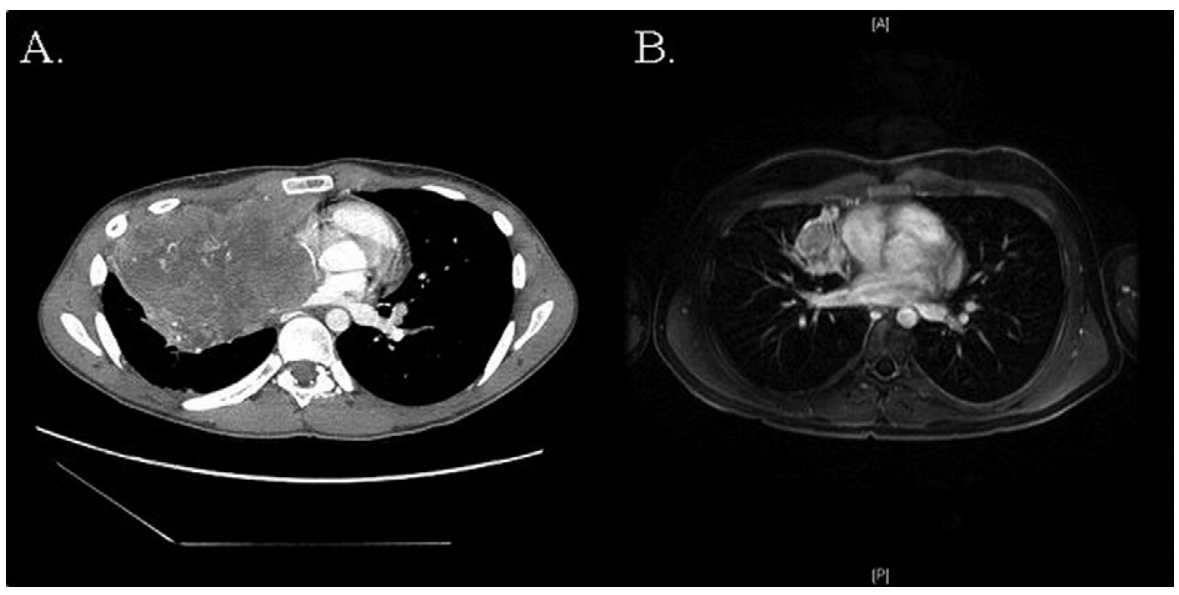

Figure 1. A, Chest CT demonstrating extensive mediastinal mass involving right and left atria. B. Chest MRI after chemotherapy showing significant reduction in tumor volume.

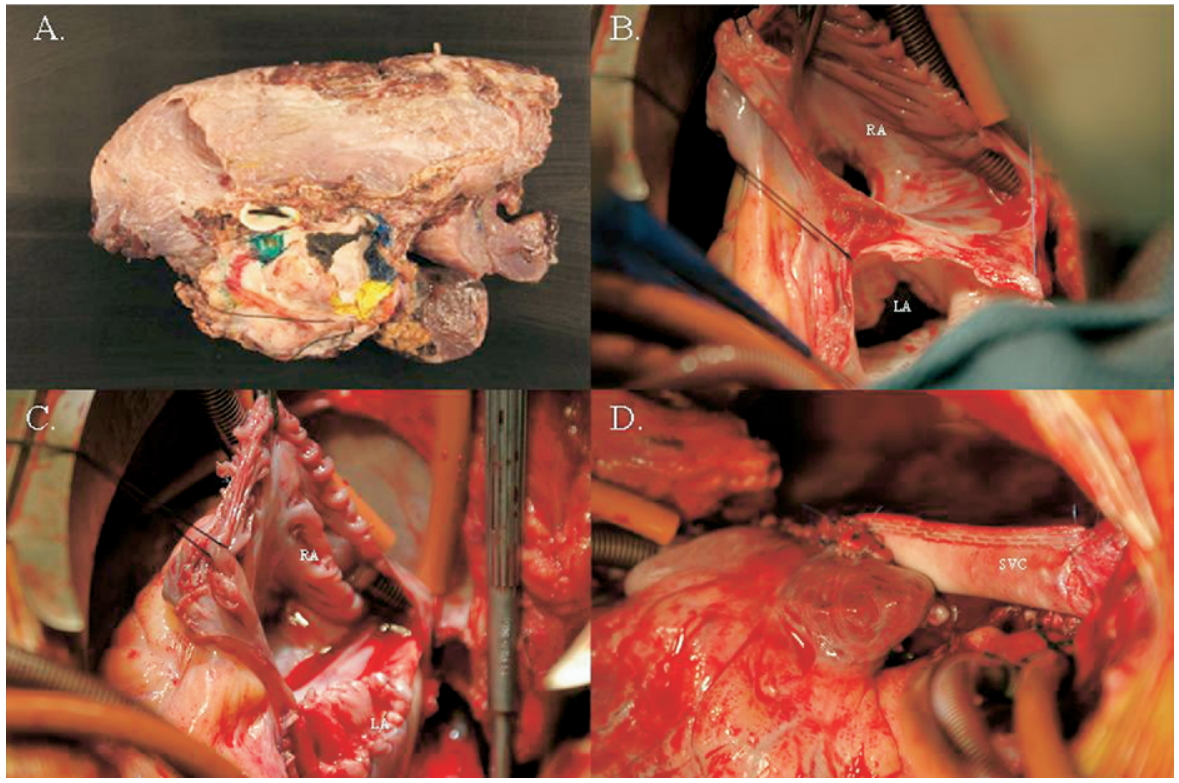

Figure 2. A, Gross pathology of mass. B, Operative photograph of left and right atrial remnants (left atrium and right atrium, respectively). C, Operative photograph of reconstructed left atrium with right atrial reconstruction ensuing. D, Operative photograph of reconstructed SVC. $L A$, Left atrium; $R A$, right atrium.

prevent cardiac herniation and to provide support to the SVC graft. The patient was weaned from bypass and the chest closed. The patient had an uneventful postoperative course and was discharged from the hospital on postoperative day 14 with a regular atrial rhythm. Final pathologic examination confirmed negative resection margins with only $40 \%$ viable tumor. The patient received further adjuvant chemoradiation therapy 6 weeks postoperatively.

\section{Discussion}

The Ewing sarcoma family of tumors, which includes Ewing sarcoma, primitive neuroectodermal tumor, neuroepithelioma, and Askin tumor, is thought to derive from neural crest cells. Genetic analysis of these tumors has revealed a translocation between chromosomes 11 and 22 that is shared by this family of sarcomas. Although typically arising from the bony skeleton, these tumors have been described to appear in almost any soft tissue in the body. ${ }^{1}$
Our case illustrates the importance of multimodality treatment in dealing with Ewing sarcoma. This patient's tumor was unresectable at initial presentation, and chemotherapy was essential in downsizing the tumor to allow resection. Furthermore, resection alone is associated with an $80 \%$ to $90 \%$ chance of metastatic disease inasmuch as these tumors are highly aggressive and subclinical metastasis is assumed. ${ }^{1}$ Hence, chemotherapy up front not only facilitates surgical resection but also improves long-term outcomes. $^{1}$

This patient's mediastinal tumor with cardiac invasion has been reported only once before in the English literature. There have been several reports of these tumors metastasizing to the heart, which were then surgically excised. ${ }^{2-4}$ In the other reported case of primary tumor in the heart, the patient underwent heart transplantation. $^{4}$ This report not only demonstrates the second case of Ewing sarcoma with cardiac involvement but also the first case of 
resection of a primary Ewing sarcoma involving the heart without cardiac transplantation.

Various techniques of SVC reconstruction have been described. We describe a technique in which a bovine pericardial tube is fashioned with a stapling device. The pericardial tube graft is widely used in surgery for congenital heart disease for procedures such as the extracardiac Fontan and caval reconstruction, but has found limited uses in surgery for malignant thoracic diseases. This technique has been reported only twice before for SVC reconstruction for malignancy but after extended pulmonary resection. ${ }^{5}$ This simple method facilitated our reconstruction.

This case illustrates the need for multimodality therapy for Ewing sarcoma delivered through a multidisciplinary approach involving thoracic and cardiac surgeons as well as oncologists.

\section{References}

1. Grier HE. The Ewing family of tumors. Ewing's sarcoma and primitive neuroectodermal tumors. Pediatr Clin North Am. 1997;44: 991-1004.

2. Flinn RM, Foyle A, Montague TJ. Extraskeletal Ewing's sarcoma with fatal cardiac metastasis. CMAJ. 1985;133:1017-8.

3. Chandramohan NK, Hussain MB, Nayak N, Kattoor J, Pandey M, Krishnankutty R. Multiple cardiac metastases from Ewing's sarcoma. Can J Cardiol. 2005;21:525-7.

4. Higgins JC, Katzman PJ, Yeager SB, Dickerman JD, Leavitt BJ, Tischler MD, et al. Extraskeletal Ewing's sarcoma of primary cardiac origin. Pediatr Cardiol. 1994;15:207-8.

5. Spaggiari L, Veronesi G, D’Aiuto M, Tosoni A. Superior vena cava reconstruction using heterologous pericardial tube after extended resection for lung cancer. Eur J Cardiothorac Surg. 2004; 26:649-51

\section{Combined pedicled antropyloroplasty and gastric pull-up reconstruction for corrosive esophagogastric stricture}

Tzu-Ping Chen, MD, Chi-Hsiao Yeh, MD, PhD, Yi-Cheng Wu, MD, and Hui-Ping Liu, MD, Keelung, Taiwan

Supplemental material is available online.

\section{Clinical Summary}

A 40-year-old woman with a history of depression was treated at our hospital for esophageal stricture resulting from ingestion of an alkaline substance. Endoscopic examination revealed a circumferential stricture $22 \mathrm{~cm}$ from the incisors, extending to the distal esophagus. A feeding jejunostomy was created for initial nutritional support. In the 3 months after the injury, the patient received esophageal balloon dilatation on 3 occasions. Contrast meal studies revealed long-segment stricture from the carina to the esophagogastic junction and distal antrum resulting in partial gastric outlet obstruction (Figure 1). After failure of endoscopic dilatation, she was subsequently scheduled for surgery owing to progressive vomiting and swallowing difficulty.

From the Division of Thoracic and Cardiovascular Surgery, Chang Gung Memorial Hospital, Keelung, Taiwan.

Received for publication Jan 30, 2007; accepted for publication Feb 7 , 2007.

Address for reprints: Dr. Tzu-Ping Chen, Division of Thoracic and Cardiovascular Surgery, Chang Gung Memorial Hospital, 222 Mai-Cin Road, Keelung 204, Taiwan (E-mail: kk13490@yahoo.com.tw).

J Thorac Cardiovasc Surg 2007;133:1669-71

$0022-5223 / \$ 32.00$

Copyright $\odot 2007$ by The American Association for Thoracic Surgery doi:10.1016/j.jtcvs.2007.02.006

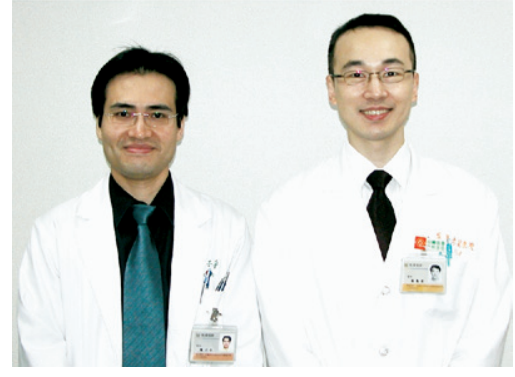

Drs Chen and Yeh (left to right)

Esophagectomy was performed via video-assisted thoracic surgery with the patient under general anesthesia. An upper midline laparotomy was then performed. The gastrocolic and gastrosplenic ligaments were dissected, and the short gastric vessels were divided. The hepatogastric ligament was then opened, and the left gastric artery and vein were doubly ligated and cut. After a Kocher maneuver, the stomach was fully mobilized and transected between the points of three fourths of the lesser curvature and the lateral gastroesophageal junction with 3 Autosuture staples (GIA75; Ethicon Endosurgery, Cincinnati, Ohio). On closer inspection, there was fibrotic scarring in the distorted region of antrum/pylorus, which was contracted and rigid. Palpation of this area revealed 5-cm segmental thickening in the gastric outlet region. The lesser curvature was divided at one third from the pylorus, and a $5 \times 5-\mathrm{cm}$ pedicled flap was created based on the right gastric artery (Figure 2). After the scarred antrum/pylorus was opened longitudinally, the gastric flap was attached to the antrotomy with a double-layer hand-sewn approximation (Figures 2 and E1). The staple line along the lesser curvature was oversewn with a continuous layer of absorbable suture. Esophagogastrostomy was accomplished by gastric pull-up of the left side of the neck through the posterior mediastinal route. Subsequent contrast studies revealed a patent cervical anastomosis and adequate pyloric drainage (Figure 1). The patient was able to resume solid and liquid oral intake from postoperative day 9. At 24 months' follow-up, satisfactory body weight gain was noted. 